\title{
Expression of Bacillus subtilis neutral protease gene (nprE) in Saccharomyces cerevisiae
}

\author{
LIN-FA WANG $\dagger$ and RodNeY J. DevenISH*
}

Department of Biochemistry, and the Centre for Molecular Biology and Medicine, Monash University, Clayton, Victoria 3168, Australia

(Received 10 August 1992; accepted 19 October 1992)

\begin{abstract}
Expression in the yeast Saccharomyces cerevisiae of the intact $n p r E$ gene of Bacillus subtilis, which encodes the pre-pro-NprE neutral protease precursor, resulted in intracellular accumulation of unprocessed precursor without detectable secretion or processing of the expressed gene product. When sequences specifying the signal peptide of yeast invertase were fused upstream of sequences encoding the mature $\mathrm{NprE}$ enzyme, $n p r E$ gene products were secreted into the culture medium. The secreted protein products were, however, highly glycosylated and biologically inactive.
\end{abstract}

\section{Introduction}

The $\alpha$-amylase genes from various sources (Filho et al., 1986; Sato et al., 1986; Rothstein et al., 1987), including Bacillus (Ruohonen et al., 1987; Nonato \& Shishido, 1988; Kovaleva et al., 1989), have been successfully expressed in Saccharomyces cerevisiae, but no work has yet been reported on the expression and secretion of heterologous, especially bacterial, protease genes in yeast. $B$. subtilis secretes a large number of extracellular proteases during the late phase of growth (Priest, 1977). All share a common structural feature, a pro-peptide located between the coding regions for signal peptide (or pre-peptide) and the mature enzyme (Stahl \& Ferrari, 1984; Yang et al., 1984; Bruckner et al., 1990; Rufo et al., 1990). The neutral protease (NprE) of B. subtilis accounts for more than $70 \%$ of total activity of all extracellular proteases, and has the largest pro-peptide of 197 amino acid residues (Yang et al., 1984).

We have recently demonstrated that the $B$. subtilis neutral protease gene ( $n p r E$ ) can be used as a reporter gene with applications in molecular biology and biotechnology (Wu et al., 1991). By removing its original ribosome binding site (RBS), we have also achieved expression of the gene in Escherichia coli such that protease is secreted from cells (Wang et al., 1990). In this

*Author for correspondence. Tel. 613565 3782; fax 6135654699.

†Present address: CSIRO, Australian Animal Health Laboratory, PO Bag 24, Geelong, Victoria 3220, Australia. communication, we report our studies on the expression and secretion of the B. subtilis $n p r E$ gene in yeast.

\section{Methods}

Yeast strain 334 MAT $\alpha$ pep4-3 prb1-1122 ura3-52 leu2-3, 112 reg1501 gall (Hovland et al., 1989) and plasmid pLF1 (Nagley et al., 1988) were used for this study. The PCR primers used to engineer the recombinant genes are listed in Table 1. A wild-type $n p r E$ gene cassette lacking the native RBS was obtained by PCR amplification using primers P1 and P2 as described previously (Wang et al., 1990), and subcloned into the unique $B g I I I$ site of $\mathrm{pLF} 1$ to form expression vector pLF-wtNPR (Fig. 1). Similarly, the SphI-BamHI fragment encoding the mature NprE protease was obtained by PCR amplification with primers $\mathrm{P} 2$ and $\mathrm{P} 3$, and then joined to a BamHI-SphI fragment encoding the yeast invertase signal peptide (Carlson \& Botstein, 1982) obtained by direct PCR amplification of yeast chromosomal DNA with primers $\mathrm{P} 4$ and P5. The hybrid gene cassette was cloned into pLF1 to form pLF-ImNPR (Fig. 1).

Yeast transformation of plasmid DNA was carried out essentially as described by Klebe et al. (1983). For expression studies, yeast cells were grown at $30^{\circ} \mathrm{C}$ in liquid YEPD medium for $48 \mathrm{~h}$. The culture was separated into cell pellet and culture medium by centrifugation using a Sorvall SS-34 rotor at 10000 r.p.m. for $10 \mathrm{~min}$ at $4{ }^{\circ} \mathrm{C}$. The cell pellet was resuspended in $7 \mathrm{M}$-guanidine hydrochloride and then vortexed in the presence of glass beads to produce a total cell lysate. Portions of lysate equivalent to the cell pellet from $300 \mu$ l of the original culture were mixed with an equal volume of $2 \times$ Laemmli sample buffer and then subjected to SDS-PAGE $(10 \%, \mathrm{w} / \mathrm{v})$ analysis. Culture medium aliquots $(300 \mu \mathrm{l})$ were concentrated by precipitation with 4 vols cold acetone before analysis by SDS-PAGE $(10 \%, \mathrm{w} / \mathrm{v})$.

Protease assays and immunoblots were carried out as described previously (Wang et al., 1990; Wu et al., 1991). Endoglycosidase D and endo- $\alpha$ - $N$-acetylgalactosaminidase were purchased from Boehringer Mannheim Biochemica, and were used according to the supplier's instructions; $300 \mu \mathrm{l}$ culture medium fractions were incubated with $2 \mu \mathrm{l}$ 
Table 1. PCR oligonucleotide primers

\begin{tabular}{|c|c|c|c|}
\hline Primer & Sequence $\left(5^{\prime} \rightarrow 3^{\prime}\right)$ & Position* & Target gene $\dagger$ \\
\hline P1 & $\begin{array}{l}\text { CCATGGATCCAAATATAATGGGTTTAGGTAAG } \\
\text { BamHI }\end{array}$ & $2-16$ & NPR \\
\hline P2 & $\begin{array}{c}\text { GCGGCTGGATCCCTCTTTATGCAA } \\
\text { BamHI }\end{array}$ & $1676-1700$ & NPR(c) \\
\hline P3 & $\underset{S p h \mathrm{I}}{\text { CAAAATAAAGTAGCATGCGCCGCCGCCAC }}$ & $645-673$ & NPR \\
\hline P4 & $\begin{array}{l}\text { TCACGGATCCATATGATGCTTTTGCAAGC } \\
\text { BamHI }\end{array}$ & $1-17$ & IVT \\
\hline P5 & 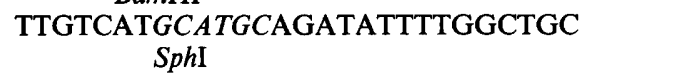 & $39-67$ & $\operatorname{IVT}(\mathrm{c})$ \\
\hline
\end{tabular}

* Nucleotide A of the ATG translational initiation codon is designated residue number 1.

$\dagger$ NPR, gene for $B$. subtilis neutral protease; IVT, gene for yeast invertase; c, complementary strand.

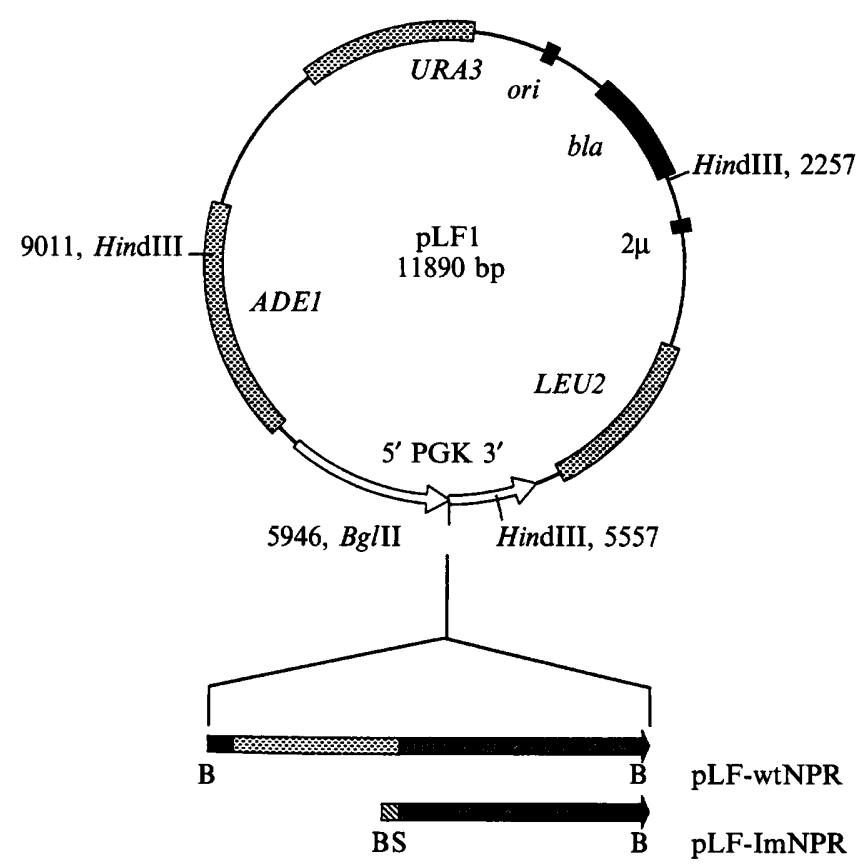

Fig. 1. Structures of recombinant plasmids constructed for the expression of the B. subtilis $n p r E$ gene in yeast. pLF1 (upper part of the figure) is a shuttle vector for expression in yeast of foreign genes under the control of yeast $P G K$ gene regulatory elements. The functional replicons, $E$. coli (ori) and yeast $(2 \mu)$, are represented by solid bars. Selectable markers used in E. coli (bla) and yeast ( $L E U 2, U R A 3$, $A D E 1)$ are indicated as shaded and dotted regions, respectively. The $5^{\prime}$ (promoter) region and the $3^{\prime}$ (transcription termination) regions of the yeast $P G K 1$ gene are shown as two open arrows separated by the unique $B g I I I$ cloning site. The lower part of the figure shows the two $B a m H I$ gene cassettes cloned in the $B g I I I$ site to create plasmids pLFwtNPR and pLF-ImNPR, respectively. The shaded region represents the mature NprE coding sequence with the arrow indicating the direction of transcription, dotted region the pro-peptide, solid region the signal peptide, and the hatched region the yeast invertase signal peptide. Abbreviations for restriction enzymes: B, BamHI; and S, SphI.

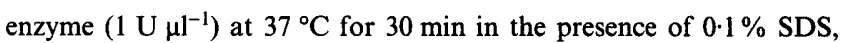
before being precipitated with 4 vols cold acetone and analysis by immunoblotting.

\section{Results and Discussion}

Extracellular proteases, like extracellular amylases, are a class of enzymes with great potential for use as reporter enzymes in studies of gene regulation and protein secretion. By comparison with methods used to assay amylases, those for protease detection are much easier, and can be carried out in situ without the necessity for staining procedures (Wang \& Doi, 1987; Wu et al., 1991).

Encouraged by the direct expression and secretion of $B$. subtilis $\alpha$-amylases in $S$. cerevisiae (Ruohonen et al., 1987; Nonato \& Shisido, 1988; Kovaleva et al., 1989) and by our recent demonstration of direct expression and secretion of $B$. subtilis neutral protease in E. coli (Wang et al., 1990) we began to test the feasibility of using the $B$. subtilis nprE gene in yeast as a reporter system. The structures of the two expression plasmids constructed in the course of this study are illustrated in Fig. 1. The sequence integrity and functionality of the PCR-derived $n p r E$ gene cassette was tested by secretion of functional protease from $E$. coli as described in our previous paper (Wang et al., 1990) before it was inserted into the vector pLF1 for expression studies in yeast. The resulting recombinant vector, pLF-wtNPR, was introduced into yeast strain 334 by PEG transformation (Klebe et al., 1987) and its presence in the resulting transformants was confirmed by PCR analysis using primers $\mathrm{P} 1$ and $\mathrm{P} 2$ with total yeast DNA as template (data not shown).

Liquid YEPD cultures were set up as described in Methods to study the expression and secretion of the $n p r E$ gene in yeast. When the culture medium from recombinant yeast strain 334[pLF-wtNPR] was analysed for protease production using either plate or liquid assay methods (Wang \& Doi, 1987; Wang et al., 1990) no significant activity was detected over the control recombinant strain 334[pLF1]. This result could have been due to any one of the following reasons: (i) failure to 


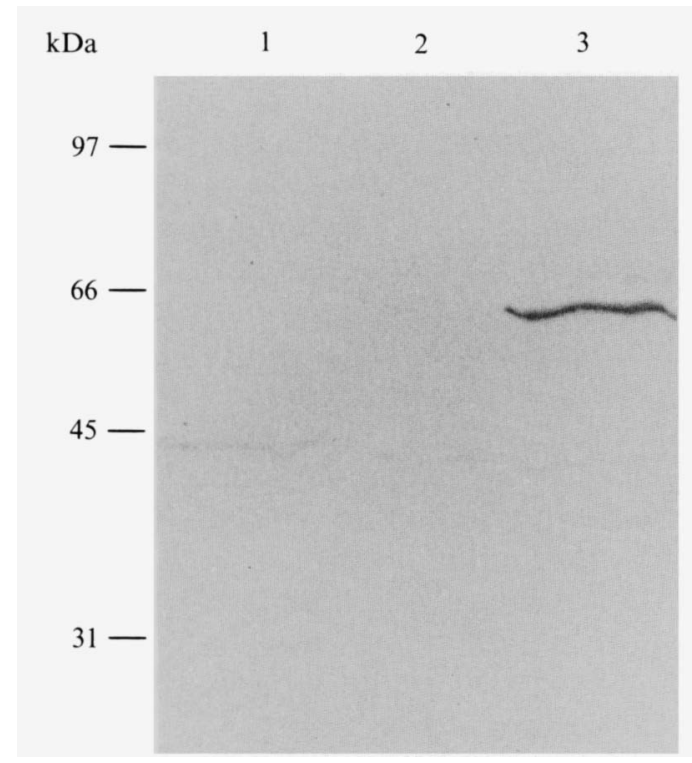

Fig. 2. Test for intracellular expression of the $n p r E$ gene in yeast made by Western blot analysis on 10\% (w/v/) SDS-PAGE. Lanes 1 to 3 display samples of total cell lysate prepared from yeast strains 334 , 334[pLF1], and 334[pLF-wtNPR], respectively. Positions of molecular weight mass markers (Rainbow, Amersham) run on the same gel are shown on the left.

express the $n p r E$ gene; (ii) the $n p r E$ gene was expressed, but the product not secreted; (iii) the gene product was expressed and secreted but was inactive owing to posttranslational modification or incorrect processing of the pre-pro-enzyme. To distinguish between these possible causes, immunoblot analysis was carried out using affinity-purified rabbit anti-NprE sera (Wang et al., 1990; Wu et al., 1991). As shown in Fig. 2, when the intracellular fraction (i.e. total cell lysate) was analysed, a specific band of about $65 \mathrm{kDa}$ was detected only for the strain carrying the functional $n p r E$ gene (lane 3), while the lower faint band (around $42 \mathrm{kDa}$ ) appeared in all lanes, indicating its probable identity as a crossreactive protein originating from the yeast host cells. The size of the NprE-related polypeptide expressed from strain $334[\mathrm{pLF}-\mathrm{wtNPR}]$ corresponds to that predicted for the unprocessed pre-pro-NprE polypeptide (Yang et al., 1984). A similar immunoblot was also performed for extracellular fractions (i.e. culture medium), but no positive signal was detected for cells carrying pLFwtNPR (data not shown). These results would suggest that the nprE gene was expressed in yeast under the control of the $P G K 1$ promoter, but that the native signal peptide failed to function as a targeting signal to direct the gene product into the yeast secretory pathway. Supporting evidence includes, first, the absence of any NprE-related polypeptide in the culture medium, and secondly, the presence of a relatively sharp band of the unprocessed pre-pro- $\mathrm{NprE}$ inside the cell. If the signal peptide of the B. subtilis neutral protease was able to

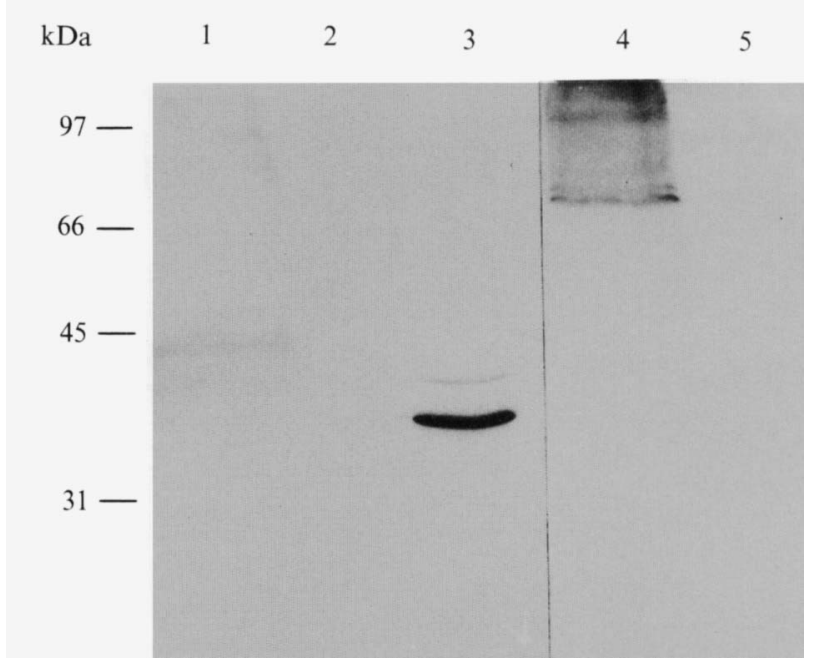

Fig. 3. Western blot analysis of $n p r E$ gene products expressed from strain 334[pLF-ImNPR]. Lanes 1 and 2: total cell lysate from strains 334[pLF-ImNPR] and 334[pLF1], respectively. Lane 3: positive control of mature NprE protease expressed from B. subtilis. Lanes 4 and 5: culture medium from strains 334[pLF-ImNPR] and 334[pLF1], respectively.

function in the initial phases of the secretion process, its gene product may still have remained inside the cell, but the polypeptide would most likely have been glycosylated (see Ruohonen et al., 1987 and later discussion in this paper), which would have resulted in the diffusion or smearing of the signals on Western blots.

The failure to achieve secretion may also have resulted from sequence features of the polypeptide rather than its signal peptide. To examine further the extent to which the mature neutral protease enzyme could be secreted from yeast, plasmid pLF-ImNPR was constructed by direct fusion of the coding sequence of the yeast invertase signal peptide immediately upstream of sequences encoding the mature NprE protease via PCR-mediated gene construction (see Methods for details). The sequence integrity of this PCR-derived hybrid gene was confirmed by direct DNA sequencing (data not shown) before the gene cassette was used in yeast expression studies.

When this hybrid gene was expressed in yeast, no protease activity was detected. However, when the intracellular and extracellular fractions were analysed by immunoblotting, it was found that the majority of the $\mathrm{NprE}$-related polypeptides were present in the culture medium, i.e. they had been secreted (Fig. 3). In addition, instead of forming a sharp band on Western blots as observed for the intracellular pre-pro-NprE expressed from $\mathrm{pLF}$-wtNPR, the signals obtained for both intracellular and extracellular NprE-polypeptides showed, in this case, extensive diffusion and smearing, indicating a high level of glycosylation of the $n p r E$ gene products. The major diffused band present in the intracellular 


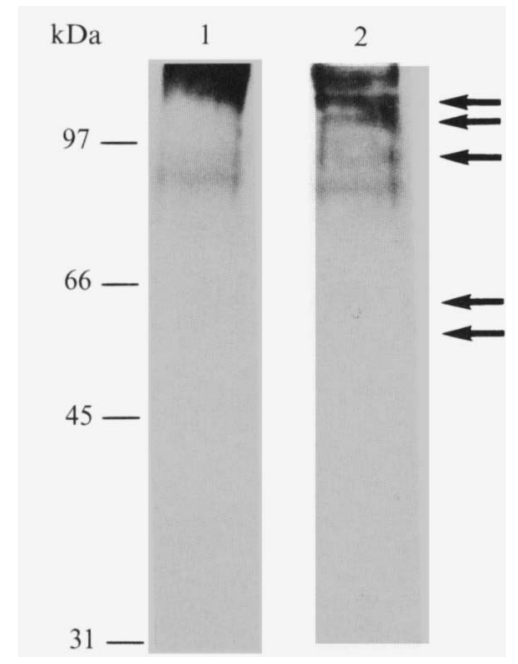

Fig. 4. Endoglycosidase D digestion of secreted $n p r E$ gene products. Western blot analysis was made of culture medium from strain $334[\mathrm{pLF}$-wtNPR], which was incubated in the presence of $0.1 \%$ SDS at $37^{\circ} \mathrm{C}$ for $30 \mathrm{~min}$ in the presence (lane 1) or absence (lane 2) of endoglycosidase $\mathrm{D}$. Arrows on the right indicate the bands unique to lane 2 .

fraction (lane 1) had a molecular mass of about $45 \mathrm{kDa}$, which is bigger than the unmodified mature neutral protease (about $38 \mathrm{kDa}$, lane 3 ) or the unmodified preNprE with the invertase signal peptide attached (about $40 \mathrm{kDa}$ ). These results indicated that the secretion process was very efficient, with all of the gene products either being secreted or having at least entered the secretion pathway as indicated by the absence of unmodified nascent pre-NprE polypeptides of the same mobility as seen in the pLF-wtNPR expression experiment (Fig. 2). These results support the contention that the failure of secretion of the native pre-pro-NprE in yeast most likely results from the failure of the native signal peptide to function in yeast rather than any incompatibility of the NprE protease with the yeast secretory pathway.

There are three potential $N$-linked glycosylation sites in the mature NprE enzyme, Asn-Ile-Ser (residues 18-20), Asn-Glu-Ser (residues 166-168), and Asn-AspThr (residues 177-179). The Ser and Thr contents of the NprE protease are $10.0 \%$ and $9.5 \%$, respectively. These features suggest that it is potentially a good substrate for $N$ - and $O$-linked glycosylation. To confirm that the $n p r E$ gene products expressed from pLF-ImNPR were actually glycosylated, supernatant samples were treated with commercially available endoglycosidases. Sharpening of the Western blot signals would have indicated degradation of the carbohydrate moieties attached to the NprE proteins. The results obtained after the treatment of supernatant fractions with endoglycosidase $D$ are shown in Fig. 4. As indicated by arrows, several bands appeared in the digested sample (lane 2) which were absent in the control sample (lane 1). No sharp band was observed in the region around $38 \mathrm{kDa}$ (the size predicted for the unmodified NprE protease), suggesting either that the endoglycosidase $\mathrm{D}$ digestion was incomplete or that more than one type of glycosylation was present on the NprE protein or both. Interestingly, digestion of samples with endo- $\alpha-N$-acetylgalactosaminidase had no effect on the mobility of NprE proteins as determined by Western blot (data not shown).

In conclusion, the results presented in this communication illustrated two important points: (i) different signal peptides, derived from the same organism $(B a-$ cillus), may behave very differently in a heterologous system (yeast); and (ii) in contrast to the $B$. amyloliquefaciens amylase, whose glycosylated form was still active (Ruohonen et al., 1987; Kovaleva et al., 1989), the glycosylated $B$. subtilis neutral protease secreted from yeast is biologically inactive.

We thank Dr R. H. Doi for providing anti-neutral protease sera, Dr R. A. Sclafani for providing yeast strain 334 and Professors P. Nagley and A.W. Linnane for providing us with a stimulating research environment in which to carry out this work. This study was supported in part by a Monash University Special Research Fund Grant (19.122.032) to L.F.W. and ARC Grant (20.122.029) to R.J.D. and L.F.W.

\section{References}

Bruckner, R., Shoseyov, O. \& DoI, R. H. (1990). Multiple active forms of a novel serine protease from Bacillus subtilis. Molecular and General Genetics 221, 486-490.

CARLSON, M. \& BotsteIn, D. (1982). Two differentially regulated mRNAs with different $5^{\prime}$ ends encode secreted and intracellular forms of yeast invertase. Cell 28, 145-154.

Filho, S. A., Galembeck, E. V., Faria, J. B. \& Frascino, A. C. S. (1986). Stable yeast transformants that secrete functional $\alpha$-amylase encoded by cloned mouse pancreatic cDNA. Biotechnology 4, 311-315.

Hovland, P., Flick, J., Johnston, M. \& Sclafani, R. A. (1989). Galactose as a gratuitous inducer of $G A L$ gene expression of yeasts growing on glucose. Gene $83,57-64$.

Klebe, R. J, Harriss, J. V., Sharp, Z. D. \& Douglas, M. G. (1983). A general method for chemically induced transformation of bacteria and yeast. Gene 25, 333-341.

KovaleVa, I. E., Novikova, L. A. \& Luzikov, V. N. (1989). Synthesis and secretion of bacterial $\alpha$-amylase by the yeast Saccharomyces cerevisiae. FEBS Letters 251, 183-186.

Nagley, P., Farrell, L. B., Gearing, D. P., Nero, D., Meltzer, S. \& DEVENISH, R. J. (1988). Assembly of functional proton-translocating ATPase complex in yeast mitochondria with cytoplasmially synthesised subunit 8 , a polypeptide normally encoded within the organelle. Proceedings of the National Academy of Sciences of the United States of America 85, 2091-2095.

Nonanto, R. V. \& SHISHIDo, K. (1988). $\alpha$-Factor-directed synthesis of Bacillus stearothermophilus $\alpha$-amylase in Saccharomyces cerevisiae. Biochemical and Biophysical Research Communications 162, 76-82.

PrIeST, F. G. (1977). Extracellular enzyme synthesis in the genus Bacillus. Bacteriology Reviews 41, 711-753.

Rothstein, S. J, Lahners, K. N., Lazurus, C. M., Baulcombe, D. C. \& GATENBY, A. A. (1987). Synthesis and secretion of wheat $\alpha$ amylase in Saccharomyces cerevisiae. Gene 55, 353-356.

Rufo, G. A., Sullivan, B. J., Sloma, A. \& Pero, J. (1990). Isolation and characterisation of a novel extracellular metalloprotease from Bacillus subtilis. Journal of Bacteriology 172, 1019-1023.

Ruohonen, L., Hackman, P., Lehtovaara, P., Knowles, J. K. C. \& KERANEN, S. (1987). Efficient secretion of Bacillus amyloliquefaciens 
$\alpha$-amylase by its own signal peptide from Saccharomyces cerevisiae host cells. Gene 59, 161-170.

Sato, T., Tsunasawa, S., Nakamura, Y., Emi, M., Sakyama, F. \& Matsubara, K. (1986). Expression of the human salivary $\alpha$-amylase gene in yeast and characterisation of the secreted protein. Gene $\mathbf{5 0}$, 247-257.

Stahl, M. L. \& Ferrari, E. (1984). Replacement of the Bacillus subtilis subtilisin structural gene with an in vitro-derived deletion mutation. Journal of Bacteriology 158, 411-418.

Wang, L. F. \& DoI, R. H. (1987). Promoter switching during development and the termination site of the $\sigma^{43}$ operon on Bacillus subtilis. Molecular and general Genetics 207, 114-119.
Wang, L. F., EkKel, S. M. \& Devenish, R. J. (1990). Expression in Esherichia coli of the Bacillus subtilis neutral protease gene (nprE) lacking its ribosome binding site. Biochemistry International 22, 1085-1093.

Wu, Z. R., QI, B. J., JiAo, R. Q., ChEN, F. D. \& WANG, L. F. (1991). Development of a novel a Bacillus subtilis cloning system employing its neutral protease as screen marker. Gene 106, 103-107.

YaNG, M. Y., FerRari, E. \& HenNer, D. J. (1984). Cloning of the neutral protease gene of Bacillus subtilis and the use of the cloned gene to create an in vitro-derived deletion mutation. Journal of Bacteriology 160, 15-21. 